\title{
Cirurgia de estrabismo ajustável no peroperatório com anestesia tópica em pacientes com orbitopatia de Graves
}

\author{
Intraoperative adjustable strabismus surgery under drop anesthesia in \\ patients with Graves' orbitopathy
}

Patrícia Grativol Costa ${ }^{1}$

Fábio Petersen Saraiva ${ }^{2}$

Suellen Tieni Shibata ${ }^{3}$

Iara Debert ${ }^{4}$

Vivian Onoda Tomikawa ${ }^{5}$

Mariza Polati ${ }^{6}$
Trabalho realizado no Departamento de Oftalmologia e Otorrinolaringologia da Faculdade de Medicina da Universidade de São Paulo - USP - São Paulo (SP) Brasil.

Pós-graduanda do Departamento de Oftalmologia e Otorrinolaringologia do Hospital das Clínicas da Faculdade de Medicina da Universidade de São Paulo USP - São Paulo (SP) - Brasil.

Pós-graduando do Departamento de Oftalmologia e Otorrinolaringologia do Hospital das Clínicas da USP - São Paulo (SP) - Brasil.

${ }^{3}$ Acadêmica de Medicina da USP - São Paulo (SP) Brasil.

Estagiária do Departamento de Motilidade Ocular Extrínsica do Hospital das Clínicas da USP - São Paulo (SP) - Brasil.

${ }^{5}$ Estagiária do Departamento de Motilidade Ocular Extrínsica do Hospital das Clínicas da USP - São Paulo (SP) - Brasil.

${ }^{6}$ Doutora, Chefe do Departamento de Motilidade Ocular Extrínsica do Hospital das Clínicas da USP - São Paulo (SP) - Brasil.

Endereço para correspondência: Patrícia Grativol Costa. Rua Oscar Freire, 1.702/38 - São Paulo (SP) CEP 05409-011

E-mail: patriciagrativol@yahoo.com.br

Recebido para publicação em 26.04.2006

Última versão recebida em 22.11.2007

Aprovação em 11.01.2008

Nota Editorial: Depois de concluída a análise do artigo sob sigilo editorial e com a anuência do Dr. Galton Carvalho Vasconcelos sobre a divulgação de seu nome como revisor, agradecemos sua participação neste processo.

\begin{tabular}{|l|}
\hline RESUMO \\
\hline Objetivo: Descrever as características clínicas pré-operatórias dos pacien- \\
tes com estrabismo secundário à orbitopatia de Graves e os resultados da \\
cirurgia com anestesia tópica e sutura ajustável. Métodos: Estudo retros- \\
pectivo realizado no Hospital das Clínicas da Universidade de São Paulo. \\
Foram pesquisados os prontuários de todos os pacientes atendidos no \\
ambulatório de estrabismo no período de março de 1994 a maio de 2004. \\
Destes, foram separados aqueles com estrabismo associado à orbitopatia \\
de Graves submetidos à cirurgia ajustável com anestesia tópica. As \\
características clínicas pré-operatórias e os resultados cirúrgicos foram \\
levantados a partir desta análise. Resultados: Foram incluídos 13 pacien- \\
tes. O tipo de desvio mais freqüentemente encontrado foi esotropia com \\
hipotropia. Em 9 pacientes modificou-se o retrocesso programado no pré- \\
operatório. Três casos necessitaram de uma segunda cirurgia. Após 6 \\
meses de seguimento, 8 dos 13 pacientes estavam ortotrópicos ou com \\
foria pequena e com algum grau de estereopsia. Conclusão: Neste estudo \\
observou-se que $62 \%$ (8/13) dos pacientes apresentavam hipotropia com \\
esotropia, provavelmente por causa do comprometimento associado do \\
reto inferior e reto medial. Nove dos 13 pacientes necessitaram de ajuste \\
no peroperatório e apenas 3 foram reoperados, indicando a importância da \\
técnica ajustável para melhor alinhamento ocular no pós-operatório, \\
possibilitando obter resultados mais satisfatórios.
\end{tabular}

Descritores: Anestesia local; Diplopia; Doença de Graves/cirurgia; Estrabismo; Visão binocular

\section{INTRODUCÃO}

A orbitopatia de Graves é caracterizada pelo espessamento dos músculos oculares extrínsecos e aumento da gordura retrobulbar ${ }^{(1-2)}$. É mais freqüente em mulheres (86\% dos casos), na faixa etária entre 40-44 e 60-64 $\operatorname{anos}^{(3)}$. Nos homens, a doença pode ser mais grave, principalmente o comprometimento dos músculos extra-oculares ${ }^{(4-5)}$.

As manifestações clínicas incluem edema das partes moles, retração palpebral, neuropatia óptica compressiva e miopatia restritiva ${ }^{(6)}$. A frequiência destes sinais varia conforme a população estudada. Estima-se que a miopatia extra-ocular restritiva ocorra em $43 \%$ dos casos e, geralmente, é bilateral e acomete múltiplos músculos ${ }^{(7-8)}$. O desvio ocular secundário a miopatia pode ser horizontal, vertical ou torcional podendo causar diplopia ou obrigar os pacientes a adotar uma posição viciosa de cabeça para alcançar a fusão ${ }^{(8)}$. 
Embora um processo auto-imune seja muito provável, o mecanismo exato do desenvolvimento da miopatia de Graves permanece desconhecido ${ }^{(7-9)}$. Na fase aguda da doença, as células da musculatura ocular extrínsica estão dissociadas pelo edema e infiltração linfocitária. A fase crônica (miopatia sequelar) é caracterizada por reação fibrótica com numerosos fibroblastos alongados entre fibras colágenas e com presença de $\operatorname{adipócitos}^{(7,9)}$.

O tratamento cirúrgico desse tipo de estrabismo apresenta resultados imprevisíveis e, às vezes, é necessário mais de um procedimento cirúrgico para correção do desvio ocular.

O objetivo deste estudo é descrever as características clínicas pré-operatórias dos pacientes com estrabismo secundário à orbitopatia de Graves e os resultados cirúrgicos da técnica com anestesia tópica e sutura ajustável peroperatória através da análise de prontuários.

\section{MÉTODOS}

Estudo retrospectivo dos prontuários de pacientes com estrabismo secundário à miopatia de Graves que freqüentaram o ambulatório de motilidade ocular extrínsica do Hospital das Clínicas da Universidade de São Paulo.

Foram incluídos 13 pacientes no período de março de 1994 a maio de 2004, sendo 8 mulheres e 5 homens, com idade que variou entre 31 e 79 anos (média de 47,5 anos). O tempo de evolução da doença tireoidiana oscilou entre 2 e 17 anos. Dos 13 pacientes, um tinha sido submetido à cirurgia de descompressão orbitária prévia e outro à radioterapia de órbita. Com relação aos antecedentes pessoais, um paciente tinha miastenia gravis e outro era portador de adenoma hipofisário associado. Nenhum paciente apresentava antecedentes familiares de doença da tireóide.

Realizou-se: avaliação da acuidade visual, medida do desvio pelo método do prisma e cobertura alternado (para longe e perto) ou Krimsky (quando a acuidade visual era inferior a 20/200 ou quando a restrição muscular não permitia a avaliação pelo método do prisma e cobertura alternado), versões e ducções, presença de anisotropia e torcicolo, e avaliação sensorial com prisma e filtro vermelho. Todos os pacientes incluídos apresentavam-se clinicamente estáveis da tireoidopatia, e sem sinais inflamatórios orbitários e oculares, há pelo menos 6 meses (miopatia sequelar) antes da indicação cirúrgica. $\mathrm{O}$ valor do ângulo do estrabismo foi avaliado pelo menos 2 vezes em consultas realizadas com intervalo mínimo de 3 meses, para confirmação da estabilidade do desvio.

A cirurgia foi sempre realizada pelo mesmo cirurgião (MP) e utilizou-se a anestesia tópica com colírio de tetracaína a $1 \%$ (Farmacotécnica Hospitalar da Divisão de Farmácia HC FMUSP). Analisaram-se a função muscular e restrições, antes do início da cirurgia, através da prova das ducções forçadas passivas. Após incisão em conjuntiva e pexia do músculo, era realizada passagem de fio absorvível (Vicryl ${ }^{\circledR} 6-0$ ) e retrocesso programado no pré-operatório (de acordo com o tipo e valor do desvio pré-cirúrgico) com sutura ajustável e em "hangback". Em seguida, com o auxílio de foco de luz (lanterna Welch Allyn ${ }^{\circledR}$ - 76600) posicionado a $50 \mathrm{~cm}$ e dirigido para a glabela do paciente, e filtro vermelho colocado em frente ao olho não fixador, pesquisava-se a ocorrência ou não de diplopia. A cirurgia realizada era modificada ou não, isto é, realizava-se o ajuste de acordo com a resposta do paciente ao teste.

Os resultados pós-operatórios são apresentados a seguir. O estudo foi aprovado pela comissão de ética para análise de projetos de pesquisa do Hospital das Clínicas da Universidade de São Paulo.

\section{RESULTADOS}

A acuidade visual variou entre 0,1 e 1,0 no olho direito e 0,5 e 1,0 no olho esquerdo (tabela de Snellen). Doze pacientes apresentaram queixa de diplopia, 9 com diplopia vertical e 3 horizontal. Apenas 1 paciente não referiu diplopia (tinha esotropia de 90 dioptrias prismáticas). As medidas dos desvios pré e pós-operatórios, assim como o resultado da prova das ducções forçadas passivas, são demonstradas na tabela 1 .

A avaliação sensorial pré-cirúrgica demonstrou que 10 pacientes tinham fusão estável na medida objetiva, 1 fusão instável e dois sem fusão.

Avaliando-se a necessidade de realizar o ajuste per-operatório, em 9 pacientes modificou-se o retrocesso programado no pré-operatório:

- Em 3 pacientes aumentou-se o retrocesso;

- Em 4 pacientes reduziu-se o retrocesso, sendo que em um destes pacientes o músculo foi novamente reinserido na sua inserção original;

- Em 2 pacientes diminuiu-se o retrocesso, porém, após a análise com o filtro vermelho, optou-se por manter o retrocesso programado anteriormente.

Em 3 pacientes foi necessária a segunda cirurgia: em dois deles (pacientes $\mathrm{n}^{\circ} 2$ e $\mathrm{n}^{\circ} 12$ ), retrocedeu-se o reto inferior contralateral ao primeiro olho operado, pois, após a primeira cirurgia, houve uma inversão do desvio. Nestes dois casos não foram necessários ajustes per-operatórios. No terceiro paciente ( $\left.\mathrm{n}^{\circ} 13\right)$, que evoluiu com diplopia vertical no pósoperatório, foi feita a correção do desvio vertical, pois na primeira cirurgia foi optado pela correção do desvio horizontal apenas. Na segunda operação, foi feito o recuo ajustável do reto inferior. O resultado cirúrgico após 6 meses foi:

- 8 pacientes estavam ortotrópicos ou com foria pequena (até 12 dioptrias prismáticas) e com algum grau de estereopsia (Titmus de 50" até 40" de arco);

- 1 paciente $\left(\mathrm{n}^{\circ} 13\right)$ ainda tinha desvio residual para longe com diplopia, para o qual foi prescrito correção prismática e obteve-se estereopsia de 40" de arco (hipotropia de 8 dioptrias prismáticas)

- 4 pacientes ficaram com desvio residual menor que 12 dioptrias prismáticas, porém, sem diplopia. 


\begin{tabular}{|c|c|c|c|c|c|c|c|}
\hline Paciente & $\begin{array}{l}\text { Desvio longe } \\
\text { pré-cirúrgico } \\
\text { (DP) }\end{array}$ & $\begin{array}{c}\text { PDF } \\
\text { pré-operatórias }\end{array}$ & $\begin{array}{c}\text { Cirurgia } \\
\text { programada } \\
\text { (em milímetros) }\end{array}$ & $\begin{array}{l}\text { Necessidade } \\
\text { de ajuste }\end{array}$ & $\begin{array}{l}\text { Desvio longe } \\
\text { pós-cirúrgico } \\
\text { (DP) }\end{array}$ & $\begin{array}{c}\text { Necessidade } \\
\text { de segunda } \\
\text { cirurgia }\end{array}$ & $\begin{array}{l}\text { Desvio longe } \\
\text { pós-segunda } \\
\text { cirurgia (DP) }\end{array}$ \\
\hline 1 & $\begin{array}{l}\text { ET30 } \\
\text { E/D4 }\end{array}$ & $\begin{array}{l}\text { AO: discreta } \\
\text { limitação abdução }\end{array}$ & $\begin{array}{l}\text { AO: RT RM } \\
4,0 \mathrm{~mm}\end{array}$ & Sim & Orto & Não & - \\
\hline 2 & $\begin{array}{l}\text { XT10 } \\
\text { D/E20 }\end{array}$ & $\begin{array}{l}\text { OD: discreta limitação } \\
\text { da elevação e do } \\
\text { abaixamento } \\
\text { OE: acentuada limitação } \\
\text { da elevação, discreta/ } \\
\text { moderada do abaixamento } \\
\text { e moderada da abdução }\end{array}$ & $\begin{array}{l}\text { OE: RT RI } \\
6,0 \mathrm{~mm}\end{array}$ & Sim & XT 6 E/D 15 & Sim & XT 4 D/E 4 \\
\hline 3 & $\begin{array}{l}\text { ET8 } \\
\text { D/E15 }\end{array}$ & $\begin{array}{l}\text { AO: acentuada } \\
\text { limitação da elevação } \\
\text { e abdução, moderada da } \\
\text { adução e abaixamento }\end{array}$ & $\begin{array}{l}\text { OE: RT RI } \\
4,0 \mathrm{~mm}\end{array}$ & Sim & $\times 4$ & Não & - \\
\hline 4 & $\begin{array}{l}\text { XT10 } \\
\text { D/E20 }\end{array}$ & $\begin{array}{l}\text { OD: discreta limitação } \\
\text { da adução e abaixamento, } \\
\text { moderada da abdução } \\
\text { OE: moderada limitação } \\
\text { da abdução, discreta } \\
\text { da adução e moderada } \\
\text { da elevação }\end{array}$ & $\begin{array}{l}\text { OE: RT RI } \\
2,0 \mathrm{~mm}\end{array}$ & Sim & Orto & Não & - \\
\hline 5 & XT35 & $\begin{array}{l}\text { Limitação moderada } \\
\text { da adução AO }\end{array}$ & $\begin{array}{l}\text { AO: RT RL } \\
9,0 \mathrm{~mm} \\
\text { e RS RM } \\
6,0 \mathrm{~mm}\end{array}$ & Não & ET 12 & Não & - \\
\hline 6 & $\begin{array}{l}\text { ET12 } \\
\text { E/D15 }\end{array}$ & $\begin{array}{l}\text { OD: discreta limitação } \\
\text { da adução e abdução, } \\
\text { moderada da elevação } \\
\text { e abaixamento } \\
\text { OE: acentuada limitação } \\
\text { da elevação e abaixamento, } \\
\text { moderada da adução e } \\
\text { abdução }\end{array}$ & $\begin{array}{l}\text { OD: RT RI } \\
3,0 \mathrm{~mm}\end{array}$ & Sim & Orto & Não & - \\
\hline 7 & $\mathrm{D} / \mathrm{E} 45$ & $\begin{array}{l}\text { OE: ancorado em } \\
\text { abaixamento }\end{array}$ & $\begin{array}{l}\text { OE: RT RI } \\
10,0 \mathrm{~mm}\end{array}$ & Sim & Orto & Não & - \\
\hline 8 & $\begin{array}{l}\text { ET2 } \\
\text { D/E30 }\end{array}$ & $\begin{array}{l}\text { AO: discreta limitação } \\
\text { da abdução } \\
\text { OE: acentuada limitação } \\
\text { da elevação }\end{array}$ & $\begin{array}{l}\text { OE: RT RI } \\
5,0 \mathrm{~mm}\end{array}$ & Não & Orto & Não & - \\
\hline 9 & D/E35 & $\begin{array}{l}\text { OE: leve limitação } \\
\text { da elevação }\end{array}$ & $\begin{array}{l}\text { OE: RT RI } \\
5,0 \mathrm{~mm}\end{array}$ & Sim & Orto & Não & - \\
\hline 10 & $\begin{array}{l}\text { ET6 } \\
\text { D/E50 }\end{array}$ & $\begin{array}{l}\text { OD: limitação moderada } \\
\text { da elevação e discreta } \\
\text { da adução } \\
\text { OE: limitação acentuada } \\
\text { da elevação e discreta } \\
\text { da adução }\end{array}$ & $\begin{array}{l}\text { OE: RT RI } \\
8,0 \mathrm{~mm}\end{array}$ & Não & Orto & Não & - \\
\hline 11 & $\begin{array}{l}\text { ET90 } \\
\text { D/E15 }\end{array}$ & $\begin{array}{l}\text { OD: acentuada limitação } \\
\text { da abdução } \\
\text { OE: moderada limitação } \\
\text { da elevação e discreta } \\
\text { da abdução }\end{array}$ & $\begin{array}{l}\text { OE: RT RI } \\
6,0 \mathrm{~mm} \\
\text { OD: RT RM } \\
8,0 \mathrm{~mm}\end{array}$ & Não & $\mathrm{D} / \mathrm{E} 6$ & Não & - \\
\hline 12 & $\begin{array}{l}\text { ET6 } \\
\text { D/E15 }\end{array}$ & $\begin{array}{l}\text { OE: moderada/acentuada } \\
\text { limitação da elevação }\end{array}$ & $\begin{array}{l}\text { OE: RT RI } \\
4,0 \mathrm{~mm}\end{array}$ & Sim & ET 6 E/D 10 & Sim & $\mathrm{D} / \mathrm{E} 4$ \\
\hline 13 & $\begin{array}{l}\text { ET25 } \\
\text { D/E8 }\end{array}$ & $\begin{array}{l}\text { AO: acentuada limitação } \\
\text { da abdução }\end{array}$ & $\begin{array}{l}\text { AO: RT RM } \\
5,0 \mathrm{~mm}\end{array}$ & Sim & D/E 15 & Sim & X 4 D/E 8 \\
\hline
\end{tabular}




\section{DISCUSS ÃO}

A orbitopatia de Graves é a causa mais comum de estrabismo adquirido em adultos de meia idade e idosos e os problemas relacionados incluem diplopia e posição viciosa de cabeça compensatória ${ }^{(10)}$.

O músculo reto inferior é o mais comumente afetado na doença da tireóide, seguido pelo medial, superior e lateral ${ }^{(8,10-11)}$. Neste estudo observou-se que $62 \%$ (8/13) dos pacientes apresentavam hipotropia com esotropia, provavelmente por causa do comprometimento associado do reto inferior e reto medial. Outro aspecto que corrobora com este achado é a prova das ducções forçadas passivas. Dez dos 13 pacientes apresentavam limitação da elevação $(76,9 \%)$ e 8 apresentavam limitação da abdução $(61,5 \%)$. É provável que este déficit de elevação e abdução ocorra devido à restrição dos músculos antagonistas envolvidos (inferior e medial) secundário à fibrose.

Descreve-se na literatura que a cirurgia de descompressão orbitária torna a correção do estrabismo mais complexa, sendo mais comum a necessidade de reoperação ${ }^{(8,11)}$. Flanders et $\mathrm{al}^{(8)}$ relata, em sua série de casos, que 4 dos 5 pacientes que realizaram descompressão orbitária prévia necessitaram reoperar o estrabismo. Por outro lado, apenas 2 dos 17 pacientes não submetidos à descompressão prévia, foram reoperados. Em nosso estudo, apenas 1 paciente fez cirurgia de descompressão orbitária, e somente uma cirurgia foi realizada neste paciente para corrigir o estrabismo.

Vários trabalhos na literatura citam a associação da orbitopatia de Graves e miastenia gravis ${ }^{(12-14)}$. Ambas são doenças auto-imunes e a miastenia é mais freqüente em pacientes com doença da tireóide. Apresentamos um paciente ( $\left.\mathrm{n}^{\circ} 5\right)$ que além do quadro de orbitopatia de Graves, também tinha o diagnóstico de miastenia gravis controlado. Apresentava exotropia, o que coincide com o estudo de Vargas e colaboradores que citam a exotropia como um sinal de miastenia gravis em pacientes com orbitopatia de Graves ${ }^{(12)}$.

Para indicar a cirurgia para a correção do estrabismo na orbitopatia de Graves, deve-se aguardar a estabilização do desvio e o completo desaparecimento dos sinais inflamatórios oculares e orbitários ${ }^{(10,15)}$. No período de evolução da doença, outras modalidades de tratamento são utilizados, como corticosteróides, drogas imunossupressoras e radioterapia. Neste estudo, um paciente foi submetido à radioterapia prévia $\left(\mathrm{n}^{\circ} 3\right)$, sem melhora do quadro de diplopia. Outros estudos ${ }^{(16-17)}$ relatam que o uso da radioterapia isolada mostrou-se ineficaz no tratamento da miopatia restritiva e na melhora da diplopia. Embora o desvio seja muito variável na fase inflamatória, quando ocorre diplopia recorre-se ao tratamento provisório com prismas (Fresnel), oclusão e toxina botulínica ${ }^{(2,15)}$.

Kraus et al. ${ }^{(18)}$ mostra em seu estudo que a cirurgia ajustável tem como objetivo prevenir hiper ou hipocorreções. Esta técnica permite obter melhor alinhamento ocular inicial e baixos níveis de novas intervenções cirúrgicas ${ }^{(18-20)}$. Segundo Kal- padakis, a cirurgia ajustável com anestesia tópica evitou hipercorreções $(9,3 \%$ dos casos) em seu estudo de 64 pacientes e apenas um pequeno grupo apresentou diplopia no pósoperatório $(10,9 \% \text { dos casos })^{(21)}$. Em um relato com 5 pacientes operados de retrocesso do reto inferior com sutura ajustável e anestesia tópica, também se obteve resultados satisfatórios com redução do desvio e melhora da diplopia ${ }^{(15)}$.

Em nosso trabalho, 69,2\% dos pacientes necessitaram de ajuste no peroperatório ( 9 de 13 pacientes). Apenas 3 pacientes foram reoperados, sendo 2 por hipercorreção $(15,4 \%$ dos casos), o que é semelhante à literatura, que mostra taxas de reoperação do estrabismo entre 17 e $45 \%{ }^{(2)}$. Após 6 meses, todos os pacientes operados tiveram melhora da diplopia, sendo que apenas em um deles adaptou-se prisma para corrigir desvio residual.

As complicações pós-operatórias relatadas são hipo ou hipercorreção, inflamação exacerbada no pós-operatório, afrouxamento da sutura muscular, retração da pálpebra inferior, ciclotropia e variação alfabética em " $\mathrm{A}$ ”(8,15). Na nossa casuística apenas 3 pacientes necessitaram de nova correção cirúrgica. As demais complicações relatadas na literatura não foram encontradas em nosso estudo.

Não encontramos dados na literatura que comparem os resultados da técnica de sutura ajustável e anestesia tópica com as demais técnicas cirúrgicas (com anestesia geral e/ou sutura não ajustável) no tratamento da miopatia restritiva da orbitopatia de Graves. Estudo prospectivo e randomizado se faz necessário para comparar as técnicas cirúrgicas e melhor definir quais condições pré-operatórias podem influenciar no resultado cirúrgico ${ }^{(18)}$. Apesar da difícil abordagem do estrabismo na orbitopatia de Graves, observamos que a correção do desvio ocular, quando já estável, com anestesia tópica e cirurgia ajustável, pode ser uma opção no tratamento desta miopatia.

\section{ABSTRACT}

Purpose: To report the clinical features of strabismus associated with Graves' orbitopathy, and the results of surgery with adjustable suture under drop anesthesia. Methods: The charts of 13 patients who had surgical treatment for strabismus related to Graves' orbitopathy at Hospital das Clínicas of University of São Paulo were retrospectively reviewed. Ocular motility, sensorial examination and the follow-up after strabismus correction were studied. Results: Preoperatively, the most common type of deviation was esotropia with hypotropia. Adjustable recession was done in nine patients and a second surgery occurred in 3 patients. After follow-up of at least six months, 8 of 13 patients were orthotropic or had a small phoria with some degree of binocular vision. Conclusion: In this study, $62 \%(8 / 13)$ of patients showed hypotropia with esotropia, probably because fibrotic and restrictive muscles (medial and inferior rectus). In nine of 13 patients adjustable recession with a good postoperative alignment of the eyes was performed. Adjustment of strabismus surgery under drop 
anesthesia in patients with Graves' orbitopathy was successful in restoring binocular vision with minimum complications.

Keywords: Anesthesia, local; Diplopia; Graves' disease/surgery; Strabismus; Vision, binocular

\section{REFERÊNCIAS}

1. Mourits MP, Prummel MF, Wiersinga WM, Koornneef L. Clinical activity score as a guide in the management of patients with Graves' ophthalmophathy. Clin Endocrinol (Oxf). 1997;47(1):9-14. Erratum in: Clin Endocrinol (Oxf). 1997;47(5):632.

2. Coats DK, Paysse EA, Plager DA, Wallace DK. Early strabismus surgery for thyroid ophthalmopathy. Ophthalmology. 1999;106(2):324-9.

3. Brain R. Pathogenesis and treatment of endocrine exophthalmos. Lancet. 1959;1(7064):109-15.

4. Bartley GB, Fatourechi V, Kadrmas EF, Jacobsen SJ, Ilstrup DM, Garrity JA Gorman CA. The incidence of Graves' ophthalmopathy in Olmsted County, Minnesota. Am J Ophthalmol. 1995;120(4):511-7.

5. Kendler DL, Lippa J, Rootman J. The initial clinical characteristics of Graves' orbitopathy vary with age and sex. Arch Ophthalmol. 1993;111(2):197-201.

6. Shih MJ, Liao SL, Lu HY. A single transcutaneous injection with Botox for dysthyroid lid retraction. Eye. 2004;18(5):466-9.

7. Monteiro MLR. Oftalmopatia de Graves. In: Dantas AM, Monteiro MLR. Doenças da órbita. Rio de Janeiro: Cultura Médica; 2002. p.179-99.

8. Flanders M, Hastings M. Diagnosis and surgical management of strabismus associated with thyroid-related orbitopathy. J Pediatr Ophthalmol Strabismus. 1997;34(6):333-40.

9. Boschi A, Daumerie Ch, Spiritus M, Beguin C, Senou M, Yuksel D, et al.
Quantification of cells expressing the thyrotropin receptor in extraocular muscles in thyroid associated orbitopathy. Br J Ophthalmol. 2005;89(6):724-9. Comment in: Br J Ophthalmol. 2006;90(1):124-5.

10. Yang ML, Hsu HN, Ma L, Kao LY. Surgical management of strabismus for dysthyroid ophthalmopathy. Chang Gung Med J. 2004;27(11):787-93.

11. Gilbert J, Dailey RA, Christensen LE. Characteristics and outcomes of strabismus surgery after orbital decompression for thyroid eye disease. J AAPOS. 2005;9(1):26-30.

12. Vargas ME, Warren FA, Kupersmith MJ. Exotropia as a sign of myasthenia gravis in dysthyroid ophthalmopathy. Br J Ophthalmol. 1993;77(12):822-3.

13. Yaman A, Yaman H. Ocular myasthenia gravis coincident with thyroid ophthalmopathy. Neurol India. 2003; 51(1):100-1. Comment in: Neurol India. 2003;51(2):283-4; author reply 284

14. Widjaja A, Rademaker J, Gölkel C, Holstein A, Leifke E, Wat N. [Graves ophthalmopathy and ocular myasthenia]. Ophthalmologe. 2000;97(1):38-40. German.

15. Köse S, Uretmen O, Emre S, Pamukcu K. Recession of the inferior rectus muscle under topical anesthesia in thyroid ophthalmopathy. J Pediatr Ophthalmol Strabismus. 2002;39(6):331-5.

16. Ferris JD, Dawson EL, Plowman N, Adams GG, Fells P, Lee JP. Radiotherapy in thyroid eye disease: the effect on the field of binocular single vision. J AAPOS. 2002;6(2):71-6.

17. Wilson WB, Prochoda M. Radiotherapy for thyroid orbitopathy. Effects on extraocular muscle balance. Arch Ophthalmol. 1995;113(11):1420-5.

18. Kraus DJ, Bullock JD. Treatment of thyroid ocular myopathy with adjustable and nonadjustable suture strabismus surgery. Trans Am Ophthalmol Soc. 1993;91:67-79; discussion 79-84.

19. Rosenbaum AL, Metz HS, Carlson M, Jampolsky AJ. Adjustable rectus muscle recession surgery. A follow-up study. Arch Ophthalmol. 1977;95(5):817-20.

20. Rodrigues Alves CA, Regensteiner DBW, Cardoso PJM. Uso da anestesia tópica na cirurgia de anomalias oculomotoras. Arq Bras Oftalmol. 1987;50(4):167-9.

21. Kalpadakis P, Rudolph G, Boergen KP. [Adjustment of eye muscle surgery dosage under drop anesthesia in patients with Grave's orbitopathy]. Ophthalmologe. 2002;99(12):941-5. German. 